\title{
Magnetic field enhanced cake filtration of superparamagnetic PVAc-particles
}

\author{
C. Eichholz*, M. Stolarski, V. Goertz, H. Nirschl \\ Institute of Mechanical Process Engineering, University of Karlsruhe, Germany
}

\section{A R T I C L E I N F O}

\section{Article history:}

Received 8 November 2007

Received in revised form 10 March 2008

Accepted 25 March 2008

Available online 28 March 2008

\section{Keywords:}

Bio-processing

Bio-separation

Cake filtration

Downstream processing

Magnetic fields

Magnetic separation

\begin{abstract}
A B S T R A C T
The combination of two classical separation methods, cake filtration and magnetic field driven separation, for superparamagnetic nanocomposites results in positive synergetic effects and in extension of the field of application of the cake filtration process. In inhomogeneous magnetic fields magnetic particles experience a magnetic force. Experimental results show that two different effects of the magnetic field influence the cake building process. A special configuration of the magnet system leads to a slow down of the cake built-up. Due to changes of the structure the cake itself has a higher permeability. The result is an increase of the overall filtrate mass flow and therefore an improvement of filtration kinetics.

This new process could be applied to the emerging field of biotechnology, especially in the so called downstream processing.

Since the different components (protein, DNA, etc.) of, e.g. a fermentation broth are very small in size and have similar physical properties, the extraction of the target component out of this mixture is achieved only with high effort and expenses in a multi-step process. With the use of tailor-made magnetic adsorbent particles this process chain can be reduced considerably. The surface of these magnetic beads is manipulated in a way that only the target component is adsorbed selectively. Therefore the surface functionalization has to be concerted with the target component as well as the other side components to avoid the adsorption of one of those. The following separation of the target bio-product out of the remaining mixture is then accomplished due to the magnetic properties of the adsorbent particles. This paper discusses in detail the results of magnetic filtration experiments of non-functionalized particles. Such results are required for further development of this process for industrial scale bio-production. In this work the acceleration of cake filtration due to the above mentioned magnetic field effects is shown. Based on that a theoretical approach is suggested that describes both effects individually providing reference of their interaction.
\end{abstract}

(c) 2008 Elsevier Ltd. All rights reserved.

\section{Introduction}

Economic studies claim the biotechnology to be a future key technology and anticipate vast finance interests and positive effects on the job market (PricewaterhouseCoopers, 2007; Nusser, 2007). In modern biotechnological processes the natural metabolism of an organism is used to produce complex molecules during fermentation. This enables the development of new products such as pharmaceuticals as well as the cost-effective production of known products. But beside the desired product a fermentation broth usually contains also several side products which have to be removed in cumbersome purification steps, the so called downstream processing. It mainly consists of unit operations such as chromatography, ultra-centrifugation, filtration, and many more. Up to $80 \%$ of the

\footnotetext{
* Corresponding author. Tel./fax: +497216082427/2403.

E-mail address: christian.eichholz@mvm.uni-karlsruhe.de (C. Eichholz).
}

investment and operating costs originate from this downstream processing. Against the background of increasing demands on the efficiency of production processes this particular sector requires the improvement of separation devices or the development of new approaches. One attempt is to establish hybrid processes to benefit from synergetic effects that result from the combination of different techniques. For example such effects can be observed combining filtration and magnetic separation. In the past magnetic fields have been used mainly for selective sorting of strongly and weakly or non-magnetic products in minerals industry (Svoboda, 1987). Since several decades high gradient magnetic separation (HGMS) emerges into industrial scale for the separation of low concentrated suspensions, e.g. in the wastewater industry (Franzreb and Höll, 2000). Establishing this concept in bio-separation is also subject of current research (Franzreb et al., 2006). By using highly functionalized magnetic substrate particles, which adsorb the desired product like protein, enzyme, DNA, etc. selectively, bio-product can be separated out of a mixture of non-magnetic components, e.g. bio-broth 
(Safarik et al., 2001). A wide range of these surface ligands for different target products are already in application, e.g. in diagnostics or in adsorption chromatography. HGMS is a kind of deep bed filtration with a porous, non-remanent ferromagnetic matrix similar to a steel wool network with very low filter resistances. In an external magnetic field high field gradients around the wires of the matrix induce high magnetic forces in direction of the matrix wires upon approaching magnetic particles. Non-magnetic components pass the matrix with the liquid phase without being trapped. But limited capacity of the matrix makes HGMS applicable for low concentrated feed streams only.

To use the advantages of the magnetic field also for high concentrated slurries a new hybrid separation process, the magnetic field enhanced cake filtration, has been developed at the University of Karlsruhe (Fuchs, 2005). As a classical unit operation cake filtration is present in most industrial production processes for particulate products. The design ranges from lab-scale discontinuous nutsche filters to large-scale continuous filter apparatus. Experimental and theoretical investigations show that the combination of classical cake filtration and magnetic fields results in a reduction of the overall filtration resistance. In inhomogeneous magnetic fields magnetic particles experience a magnetic force. That gives the possibility to decouple solid and liquid phase motion to decrease the rate of cake formation. This implicates a reduction of the cake layer the liquid phase has to drain. Furthermore magnetic interparticle forces excite magnetic structuring and agglomeration effects, resulting in a higher permeability of the filter cake. The new hybrid process is not restricted to one procedural principle in the cake filtration. Till now the implementation in a filter press (Stolarski et al., 2006; Eichholz et al., 2007) and a nutsche filter (Fuchs et al., 2006) could be established. The new technology offers potential to increase process efficiency in the field of nano-scale magnetic particulate product systems and in bio-separation.

Such a bio-separation process consists of the following steps: fermentation, mixing, specific adsorption, magnetic separation, washing and elution. The product-specific functionalized particles are mixed with fermentation broth. The mixing provides intense contact of the particles with the bio-broth so that mass transfer limitations are negligible. The separation of the carrier particles with the attached product depends on the properties of the carrier particle rather than on the actual bio-product properties. The waste bio-broth can be recirculated into the fermentation process, if possible, or conveyed to waste treatment. After the first magnetic separation, the particles undergo several washing steps to reach the desired purity. During washing the external magnetic field is switched off and the particles are redispersed into washing liquor. To remove the contaminated washing liquor, further magnetic separation steps are performed. To retrieve a pure solution of the final product, it is removed from the particle surface in an elution step. Depending on the product and the binding properties, this elution is realized by change of $\mathrm{pH}$, ionic strength, temperature or substitution reactions. The carrier particles are for disposal in the next separation cycle. The advantage of magnetic separation over classical chromatographic technologies is the higher capacity of the particles due to their smaller size and better product contact, maximal product recovery, minimal separation time and the reduction of unit operations at the same time. Compared with HGMS the magnetic field enhanced cake filtration features a higher cleanability of the nutsche filter and suspensions with higher solid concentrations can be handled.

The detailed understanding of the magnetic field enhanced cake filtration with superparamagnetic nanocomposites and approaches to describe the new process theoretically are necessary for an optimal design of the apparatus and the whole process. The present work contributes to this understanding to leverage the process idea to an industrial scale.

\section{Theory}

\subsection{Magnetism}

Materials can be classified into different types of magnetism. The three most important are dia-, para- and ferromagnetism (Bergmann and Schaefer, 2006). The classification is accomplished according to the susceptibility $\chi$ which describes the magnetic irritability of the substance exposed to a magnetic field. Out of these categories diamagnetism $(\chi<0)$ is the weakest phenomena. Thereby counterdirected magnetic moments in the material weaken the magnetic field in the immediate surrounding. In contrast paramagnetic $(\chi>0)$ and ferromagnetic $(\chi \gg 0)$ materials intensify the magnetic field locally and experience a force in direction of increasing field gradients. Depending on susceptibility and magnetic field strength $H$ the material is magnetized up to a certain magnetization $M$,

$M=\chi \cdot H$

While magnetization increases linear for paramagnetic materials for a wide range of magnetic field strengths, it reaches a maximum for ferromagnetic material. This maximum is referred to as saturation magnetization $M_{s}$. Another characteristic is the remaining magnetization $M_{r}$ after disapplying the magnetic field. Of special importance to the proposed process is superparamagnetism. It is characterized by its ferromagnetic properties but without remanent magnetization. Therefore primary particles have to be composed of single magnetic domains in the range of 20-30 nm (Butler and Banerjee, 1975). In particle aggregates or composite particles with incorporated primary particles, as used in this work, the magnetic domains must not interact because this would abolish superparamagnetism.

For a magnetic field the following correlation of field strength $H$ and magnetic flux density $B$ can be obtained (Eq. (2)). In literature often both values are used interchangeable to describe the magnetic field strength,

$B=\mu_{0} \cdot \mu_{r} \cdot H$

with $\mu_{0}$ the magnetic vacuum permeability and $\mu_{r}$ the permeability number of the substance inside the field. In case of vacuum the magnetic permeability number is $\mu_{r}=1$. For diamagnetics is $\mu_{r}<1$, for para-, ferro- and superparamagnetics is $\mu_{r}>1$. The magnetic volume susceptibility is equal to $\chi=\mu_{r}-1$. The resulting force on a material, respectively, particle, in a magnetic field can be described by means of the magnetization of the particle $M_{p}$, the volume of the particle $V_{p}$ and the gradient of the external field $\nabla H$,

$F_{m}=\mu_{0} \cdot V_{p} \cdot M_{p} \cdot \nabla H=\frac{1}{\mu_{0}} \cdot \chi \cdot V_{p} \cdot B \cdot \nabla B$

Transferring Eq. (3) to the induced magnetic field of magnetic particles, the magnetic interaction (magnetic potential $E_{m}$ ) between two neighboring particles with the distance $r$ can be calculated (Lee et al., 1999). Eq. (4) describes the one-dimensional approach with the assumption of constant magnetization over the whole particle volume,

$E_{m}=\frac{\pi}{144} \cdot \frac{\mu_{0} \cdot M_{p}^{2} \cdot \rho_{p}^{2} \cdot d_{p}^{6}}{r^{3}}$

with $\rho_{p}$ the particle density and $d_{p}$ the particle diameter.

Due to the magnetic potential aggregation effects free moving particles can be observed according to the modified DLVO-theory (Chin et al., 2000, 2001). Strength and geometry of the superposed magnetic field as well as shear stress on the aggregates determine essentially size and density of the formed agglomerates (Stolarski et al., 2007). 


\subsection{Filtration}

The basic principle of cake filtration is the retention of solids while the liquid phase passes through the filter due to a driving force, e.g. differential pressure. By building particle bridges on top of the pores of the filter media the solid particles are held back. Once these bridges have been established, the increasing filter cake itself takes over the function of particle retention. With increasing filter cake height also the flow resistance through the cake for the liquid phase increases. For a laminar flow of a Newton fluid through an incompressible packed bed the flow rate can be calculated by Darcy's law (Sorrentino, 2002; Stiess, 1995),

$\frac{\mathrm{d} V_{l}}{\mathrm{~d} t}=\frac{A \cdot \Delta p}{\eta \cdot R}$

with $V_{l}$ the filtrate volume, $t$ the time, $A$ the filter area, $\eta$ the dynamic viscosity of the liquid phase, $\Delta p$ the differential pressure and $R$ the flow resistance. The latter results from a series connection of filter media and filter cake resistance. From Darcy's law the typical linear equation of cake building filtration (Ruth's law) can be derived (Ruth et al., 1933). This linear function correlates the ratio $t / V_{l}$ with the filtrate volume $V_{l}$,

$\frac{t}{V_{l}}=\frac{\eta \cdot \kappa \cdot r_{c}}{2 \cdot \Delta p \cdot A^{2}} \cdot V_{l}+\frac{\eta \cdot R_{m}}{\Delta p \cdot A}=a \cdot V_{l}+b$

with the specific cake resistance $r_{c}$, the filter media resistance $R_{m}$ and the concentration coefficient $\kappa$. By experimentally determining slope $a$ and intercept $b$ the filter media resistance and the specific cake resistance can be calculated. Even though incompressibility of the filter cake cannot always be taken for granted, Darcy's and Ruth's laws give proper results also for slightly compressible cakes by using integral values for the porosity, etc.

Neglecting gravitational and buoyancy forces for laminar flow the hydrodynamic force $F_{D}$ that acts on the particles can be described by the Stokes drag force,

$F_{D}=3 \cdot \pi \cdot \eta \cdot d_{p} \cdot u$

with $u$ the relative velocity between particles and liquid phase.

\subsection{Magnetic field enhanced cake filtration}

The magnetic field enhanced cake filtration results from the combination of classical cake filtration and magnetic separation. As already mentioned two different effects lead to a change in filtration behavior (Fig. 1).

In an inhomogeneous external magnetic field the magnetic force effects the movement of the solid phase, so it moves with differential speed compared to the liquid phase. In case of counterwise orientation of differential pressure and magnetic force the uncoupling of movement leads to a slower cake formation. If the field strength is high enough even the prevention of cake formation at the beginning of a filtration process is possible. Thereby the filter media is kept free of particles and the filtrate can run through the filter media almost without resistance. Only after the outflow of the excess water the particles deposit on the filter media. The magnetic force not only has an impact on the cake built-up but also on the structure of the filter cake. Exposed to a magnetic field particles form North and South Poles and act as microscopic magnets themselves. Resulting interparticle forces upon approaching particles lead to formation of chainlike agglomerates in direction of the external magnetic field (Charles, 1988). The permeability of a cake with this channeled structure is obviously higher than a cake consisting of smaller pores, broadly distributed in size. These effects together result in a strong improvement of filtration kinetics and thus to a reduction of
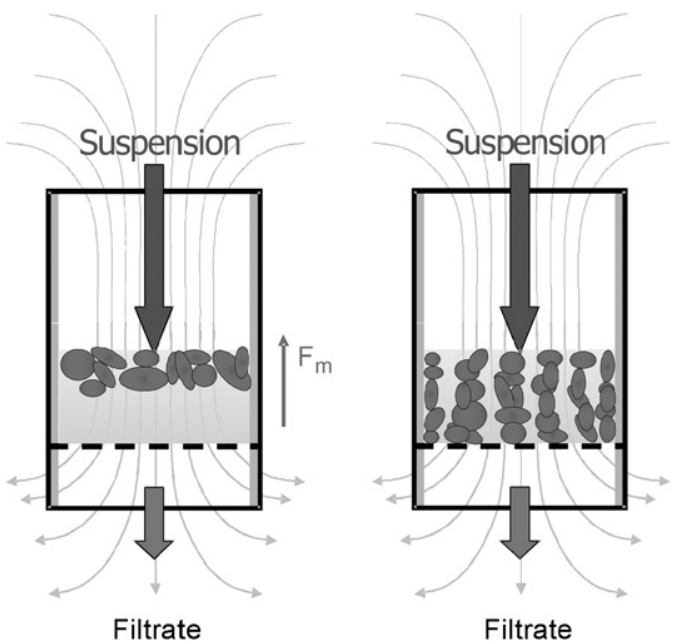

Fig. 1. Magnetic field effects: Effect of external magnetic force (left), effect of interparticulate magnetic force (right).

the filtration resistance, which can be seen in the slope of the cake building equation.

To quantify the structure of a filter cake formed in the magnetic field an energy ratio $E_{S}$ is calculated similar as Rosensweig recommends for magnetically stabilized fluidized beds (Stolarski et al., 2006; Rosensweig et al., 1981),

$E_{S}=\frac{E_{\mathrm{drag}}}{E_{m}}=\frac{3 \cdot \pi \cdot \eta \cdot d_{p}^{2} \cdot u}{1 / 144 \cdot \pi \cdot \mu_{0} \cdot \rho_{p}^{2} \cdot M_{p}^{2} \cdot d_{p}^{3}}=\frac{432 \cdot \eta \cdot u}{\mu_{0} \cdot \rho_{p}^{2} \cdot M_{p}^{2} \cdot d_{p}}$

Here the drag potential $E_{\text {drag }}$ means the energy that is needed to lift one particle against the drag force $F_{D}$ by one particle diameter (e.g. Eq. (7)). This potential describes the hindrance of the magnetically induced particle configuration due to the flow of the liquid phase. The magnetic energy is the repulsive energy between two particles in contact perpendicular to the external field direction (e.g. Eq. (4)). For $E_{S}<1$ the structure is strongly ordered, for $1<E_{S}<10$ a partly structured and for $E_{S}>10$ an unstructured bed can be assumed.

For materials with rather paramagnetic behavior Fuchs et al. (2006) calculates the change in filtration resistance by adding a magnetophoretic coefficient to the equation of cake building filtration which describes the slowdown of cake formation as a function of the external field strength (Fuchs, 2005; Fuchs et al., 2006). For ferroand superparamagnetic materials the effect of a structured filter cake plays an important role. Therefore structuring coefficients $\left(K^{*}\right.$ and $P^{*}$ ) are implemented that regard the higher overall cake porosity, originated by the high interparticulate magnetic forces (Eq. (9)). If no magnetic field is applied all coefficients are equal to 1, Eq. (9) turns into the classical equation of filtration (Eq. (6)),

$\frac{t}{V_{l}}=\frac{\eta \cdot\left[\kappa \cdot K^{*}\right] \cdot\left[r_{c} \cdot P^{*}\right]}{2 \cdot \Delta p \cdot A^{2}} \cdot\left(\frac{M_{\text {crit }} \cdot \nabla B_{\text {crit }}-M \cdot \nabla B}{M_{\text {crit }} \cdot \nabla B_{\text {crit }}}\right) \cdot V_{l}+\frac{\eta \cdot R_{m}}{\Delta p \cdot A}$

The magnetophoretic coefficient is composed of a quotient of the field force densities $M \cdot \nabla B$, which in reference to Eqs. (2) and (3) can also be described by the product $B \cdot \nabla B$. Thus the maximum impact on filtration is not in the region of maximum field strength, but in the region, where the product of field strength and field gradient form a maximum. The critical field values $M_{\text {crit }}$ and $B_{\text {crit }}$ describe the state, in which particles do not form a cake, because they are totally held back by the external magnetic force. These values can be acquired by extrapolation of experimental data or by calculations on basis of a force balance at a single particle or a particle aggregate, respectively (Fuchs, 2005; Fuchs et al., 2006). 
Since in the linear filtration equation (Eq. (6)) the concentration coefficient $\kappa$ and the specific cake resistance $r_{c}$ are functions of the porosity, two structuring coefficients $K^{*}$ and $P^{*}$ are used which compare $\kappa$ and $r_{c}$ of the magnetic field enhanced filtration with classical filtration,

$P^{*}=\frac{\phi(\varepsilon)}{\phi\left(\varepsilon_{0}\right)}$

$K^{*}=\frac{\left(1-c_{v}-\varepsilon_{0}\right)}{\left(1-c_{v}-\varepsilon\right)}$

The porosity function $\phi(\varepsilon)$ is taken from the approach of Carman and Kozeny (Sorrentino, 2002). The porosity of the filter cake is defined as a function of the applied magnetic field adapting an approach of Dubowik for columnar heterostructures (Dubowik, 1996). Thereby the particle chains are approximated as cylinders (Fig. 2). In the present case of the magnetic field enhanced cake filtration the distance $r$ between two columns is subdivided into three terms,

$$
\begin{aligned}
\varepsilon & =1-\frac{\pi}{2 \sqrt{3}}\left(\frac{d_{p}}{r}\right)^{2} \\
& =1-\frac{\pi}{2 \sqrt{3}}\left(\frac{d_{p}}{d_{p}+r_{0}+\left[\pi \cdot \mu_{0} \cdot \rho_{p}^{2} / 144 \cdot E_{\min }\right]^{1 / 3} \cdot d_{p}^{2} \cdot M^{2 / 3}}\right)^{2}
\end{aligned}
$$

The particle diameter $d_{p}$ is involved because the total distance $r$ is regarded from the middle of each column. Related to a homogeneous, randomly packed filter cake in classical filtration the overall and the cross-sectional porosity of a filter cake are identical. Selecting the cross-section as indicated in Fig. 2 as the equatorial plane of one particle layer the void fraction can also be calculated with the approach of columnar particle arrangement. Therefore an average distance has to be regarded because otherwise without superposing a magnetic field a densest sphere or column packing would result. The second term $r_{0}$ defines this average distance between the particles in classical filtration. It can be interpreted as quantity for the inhomogeneity in the filter cake built-up. The distance increases with the superposition of a magnetic field, which can be calculated from the magnetic potential (Eq. (4)). Introducing the third term the additional distance due to the repulsive magnetic potential perpendicular to the field direction is included in the calculation of the filter cake porosity. The distance increases until a minimum limit of the magnetic potential is reached. At this point the magnetic driving force is too weak to initiate further changes of the alignment. $E_{\text {min }}$ corresponds to this minimum energy limit between two columns.

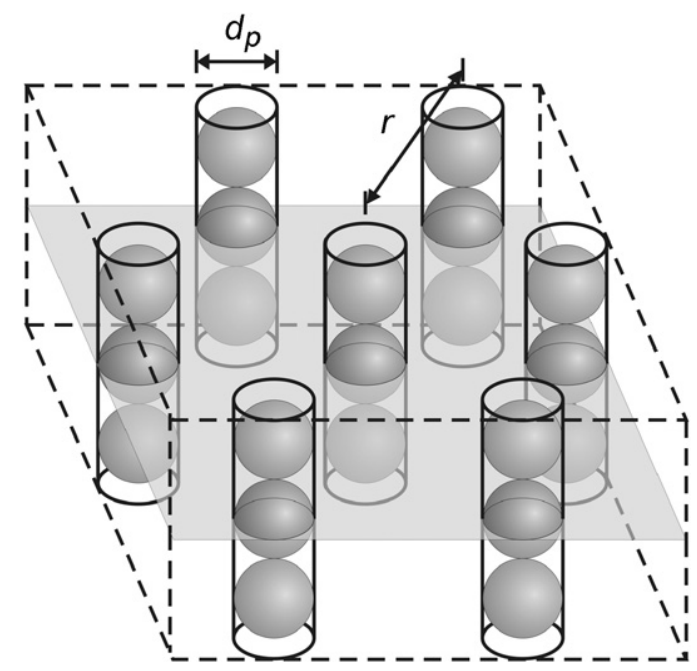

Fig. 2. Columnar-built filter cake with an equatorial plane of one particle layer.
The model of the columnar heterostructures can be applied to the whole filter cake only for higher field intensities because then magnetic interactions are predominant. For lower field strengths initially the hydrodynamic forces outweigh inhibiting the formation of these structures. Only after the slow down of filtrate flow the influence of the magnetic interactions increases and allows a structuring of the filter cake. Thus a differential regard over the cake height with the actual flow velocity is more appropriate. But the integral view should be sufficient in the present work.

The superparamagnetic character of the nanocomposites matters only after deactivating the magnetic field. Particles directly loose their magnetization and do not form magnetically induced aggregates, which is of extraordinary importance for the integration of the filtration step in the bio-separation process with a recirculation of the particles.

\section{Methods and materials}

Fig. 3 shows the scheme of the experimental apparatus. A nutsche filter (CUNO Tri-47, Cuno Filtration, Germany) with a filtration area of $10.81 \mathrm{~cm}^{2}$ is immerged in the bore (diameter $123 \mathrm{~mm}$ ) of a solenoid. The filter media (Filtryl Z-1500-K015, ZBF, Germany) has an average pore size of $15 \mu \mathrm{m}$. The pressure can be adjusted by a regulator. All parts of the filter cell are non-magnetic to not influence the magnetic field. The filtrate mass signal of the scale is recorded versus time by software. The electro magnet (Steinert Elektromagnetbau $\mathrm{GmbH}$, Germany) has maximal field strength of $0.4 \mathrm{~T}$, an iron frame bundles the magnetic field inside the bore. The filter cell is located below the horizontal center of the solenoid in a way that the highest magnetic force density $B \cdot \nabla B$ occurs in the region of the filter media. In this position magnetic and pressure forces are acting in opposite directions. In the experiments the particular magnetic field strength is applied directly before the differential pressure. Prior to each experiment the suspension $\left(c_{v}=4 \mathrm{v} / \mathrm{v} \%\right)$ is ultrasonicated to break up particle aggregations.

The magnetic field distribution inside the solenoid is measured consistently with a Hall sensor and calculated with the software QuickField $^{\mathrm{TM}}$ (Tera Analysis Ltd., Denmark). The actual value of the applied magnetic field is the one effective in the center of the filter media obtained by the software.

For the experiments magnetite seeded polyvinyl acetate particles (PVAc) are used without surface functionalization. These superparamagnetic nanocomposites are generated in a two-step process. The nano-scaled magnetite is produced in a precipitation reaction of iron-(II)-, iron-(III)-chloride salts and ammonia. The iron oxide is then incorporated into the polymer particles in a radical polymerization. Functional groups on the surface of the polymer particles can

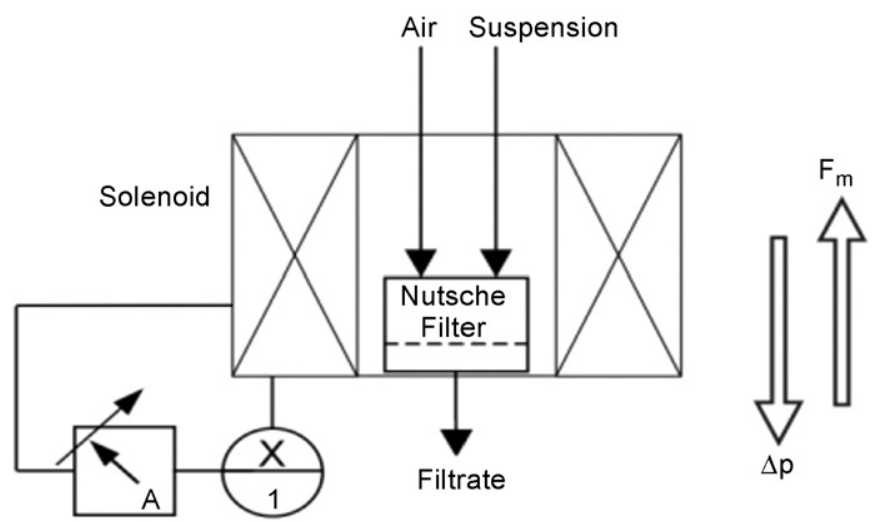

Fig. 3. Schema of the filtration apparatus. 


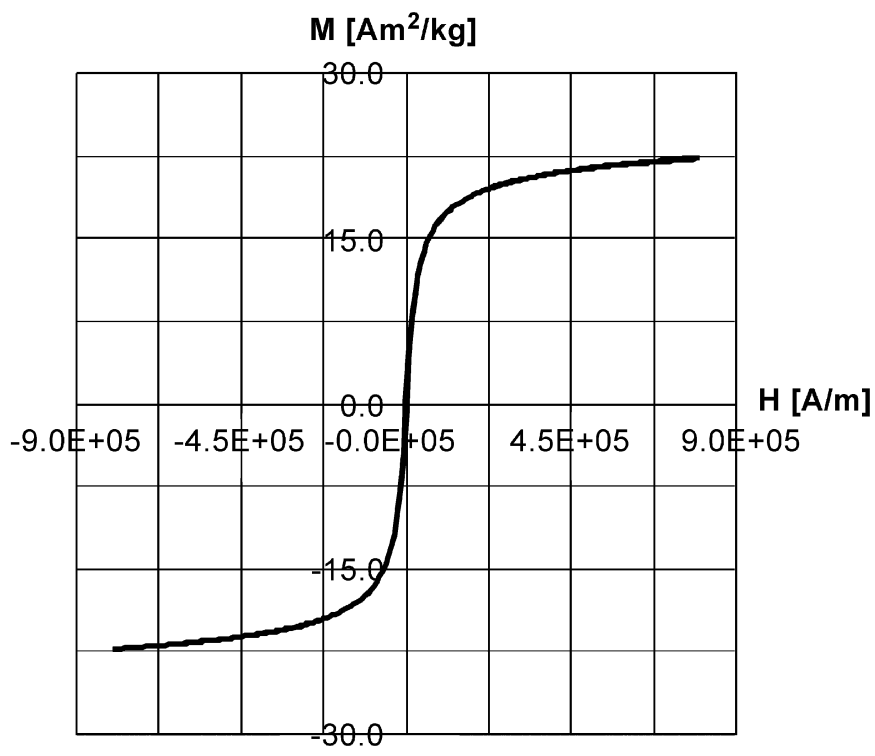

Fig. 4. Magnetization curve of the superparamagnetic PVAc-beads.

Table 1

Product properties of the magnetic beads

\begin{tabular}{lllll}
\hline Particle size & Density & Magnetization & Potential \\
\cline { 3 - 4 }$d_{50}(\mu \mathrm{m})$ & $\rho\left(\mathrm{g} / \mathrm{cm}^{3}\right)$ & $M_{S}\left(\mathrm{Am}^{2} / \mathrm{kg}\right)$ & $M_{r}\left(\mathrm{Am}^{2} / \mathrm{kg}\right)$ & $\xi$ (slurry) $(\mathrm{mV})$ \\
\hline 4.49 & 1.47 & 22.56 & 0 & -36 \\
\hline
\end{tabular}

be used to add product-specific functionalization for the selective adsorption in the bio-separation process, even though this was not followed up in the present work. The superparamagnetic character of the particles is proven by the measurement of the magnetization curve (Fig. 4). In this curve no hysteresis is observed. The beads do not have a remanent magnetization and do not agglomerate after turning off the magnetic field. This has advantage in adsorption and elution steps in bio-separation as well as in the reusability of the particles in further cycles. The exact properties of the magnetic beads are listed in Table 1.

\section{Results}

The positive effect of the superposed magnetic field can be seen by the reduction of filtration time in Figs. 5 and 6 , which show different filtrate mass signals versus time for 0.6 and 1.8 bar. As can be expected for the filtration of solids that do not form a filter cake with strong compressibility, the process is much faster applying the high pressure. The improvement of filtration kinetics results from the external magnetic force as well as from the interparticulate magnetic forces as explained extensively in the first chapters. Both effects can be determined in the filtrate curves.

The black curve represents the classical filtration without a superposed magnetic field. With increasing field strength the process is accelerated. But on closer examination of the specific runs it is observed that for both filtration pressures the filtrate curves for the lowest field strength $(0.0054 \mathrm{~T})$ do not differ in the first $10-20 \mathrm{~s}$ from classical filtration. At this time the magnetic field has no impact on the filtration process because hydrodynamic forces determine the whole process and impede a structuring of the filter cake. Accordingly the deposit in the filter media is compact, which causes a slow down of the liquid phase flow. At a certain point the velocity is sufficiently low that the magnetic interparticle forces lead to a cake

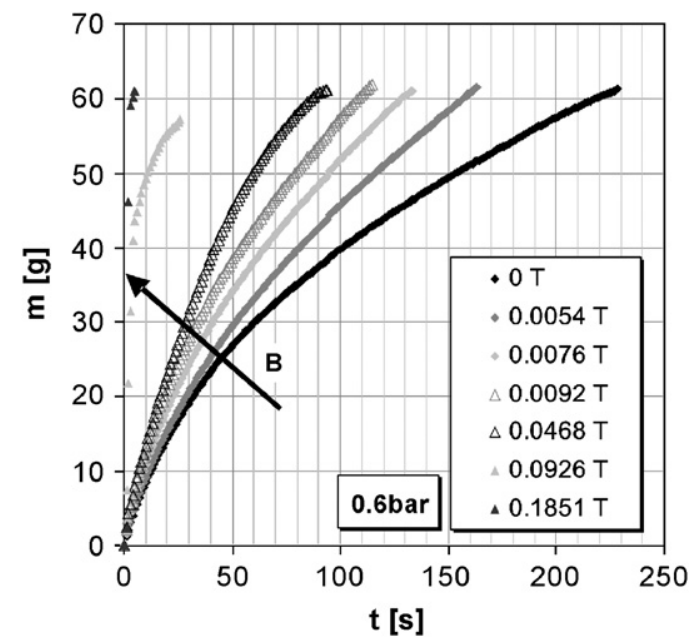

Fig. 5. Filtrate mass signals for different flux densities for 0.6 bar.

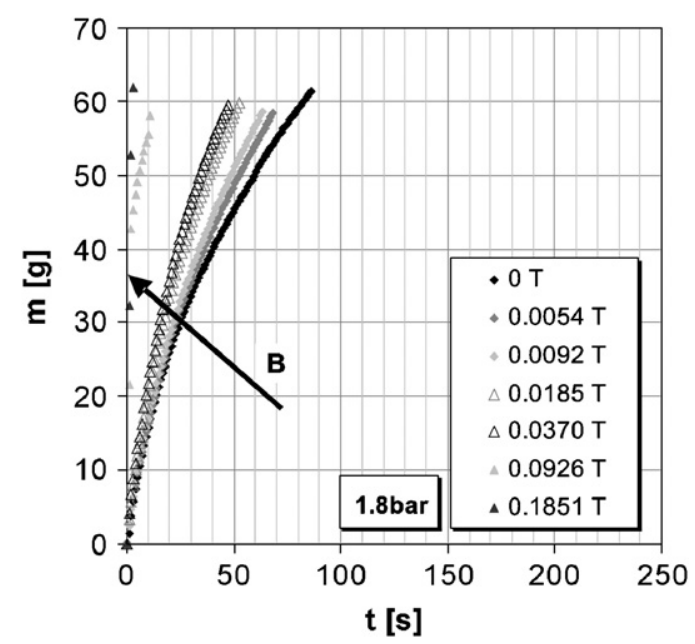

Fig. 6. Filtrate mass signals for different flux densities for 1.8 bar.

with a slightly higher permeability identifiable by the higher filtration velocity beyond. For higher field strengths the influence of the magnetic field is predominantly from the beginning.

At $0.0926 \mathrm{~T}$, which is in the region of the critical flux density defined before (Eq. (9)), the particles nearly are in a balance of forces and just a few particles are deposited on the membrane. The accumulation of the filtrate occurs abruptly with a small deceleration. Only at the end of the run the typical descent of the filtrate flow indicates a small cake formation. For higher field strengths beyond this point, here for $0.185 \mathrm{~T}$, the cake built-up is prevented totally-although not only because of axially but also because of radially acting magnetic forces. This means that the particles are not only lifted up opposed to the flow direction but also attracted to the side of the nutsche filter. Both way the filtration area is kept free of solids and the filtration can be accelerated by a factor of 60 for 0.6 bar ( 30 for 1.8 bar). Due to the used magnet system the already mentioned radial field gradient gains influence for field strengths higher than $0.0554 \mathrm{~T}$. In the further evaluations these experiments cannot be analyzed because no homogeneous filter cake is formed.

The conclusions from Figs. 5 and 6 are confirmed by the calculation of the appropriate structure parameters $E_{S}$ (Eq. (8)). The flow velocity is calculated from the filtrate flow based on the free filter cross-section. Although the parameter tends to be 


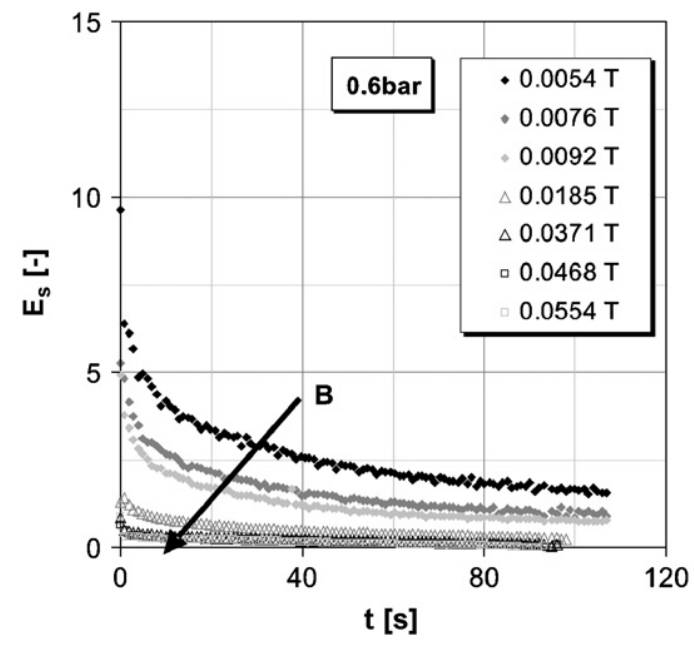

Fig. 7. Structure parameter $E_{S}$ as a function of filtration time $\mathrm{t}$ for different flux densities for 0.6 bar.

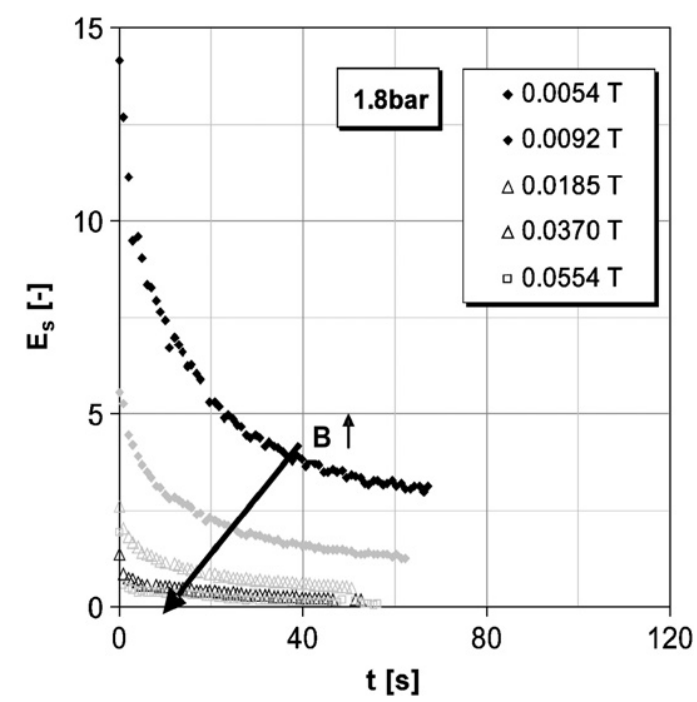

Fig. 8. Structure parameter $E_{S}$ as a function of filtration time $t$ for different flux densities for 1.8 bar.

underestimated this way the evaluation gives an idea of the structuring effects. Within the filter cake the primary particle diameter is assumed for calculations not regarding possible agglomeration effects. Figs. 7 and 8 show the development of $E_{S}$ with the duration of filtration for different magnetic field strengths and differential pressures. Throughout all field strengths $E_{S}$ is higher for 1.8 bar compared to 0.6 bar. That means that magnetic structuring effects occur especially for lower filtration pressures or hydrodynamic forces, respectively, because the alignment of the particles is hindered less. As stated before for $0.0054 \mathrm{~T}$ the first $10-20 \mathrm{~s}$ of the filtrate flows are identical with classical filtration. After this periods the magnitude of $E_{S}$ is about the same as for the next higher field strengths where the change in structure dominates the process right from the beginning. Roughly with the fall below $E_{S}=5$ the structuring effect in the filter cake can be detected in the filtrate curves (Figs. 5 and 6).

Furthermore with increasing magnetic field both diagrams reveal a decreasing variation of $E_{S}$ within one curve and between the different structure parameters. Thus it exists a limit of maximum structuring or maximum permeability. This is even more obviously comparing the initial performance of both differential pressures,

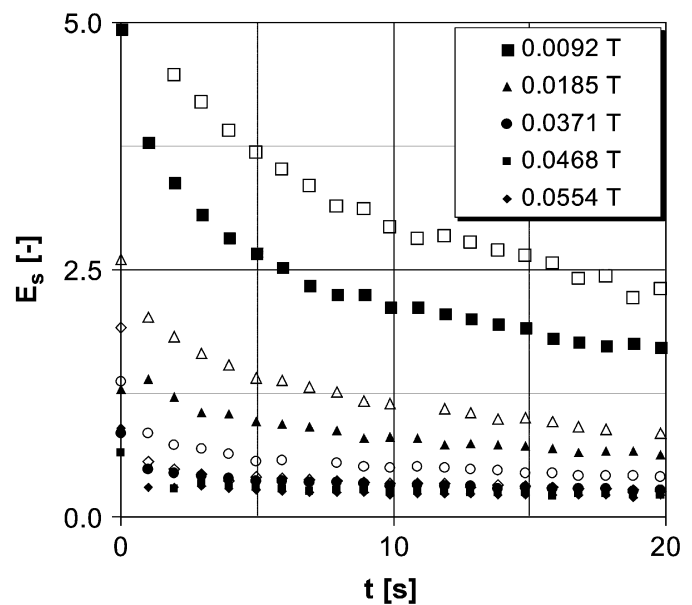

Fig. 9. Comparison of the structure parameter $E_{S}$ at the beginning of the filtration process for 0.6 bar (full symbols) and 1.8 bar (open symbols).

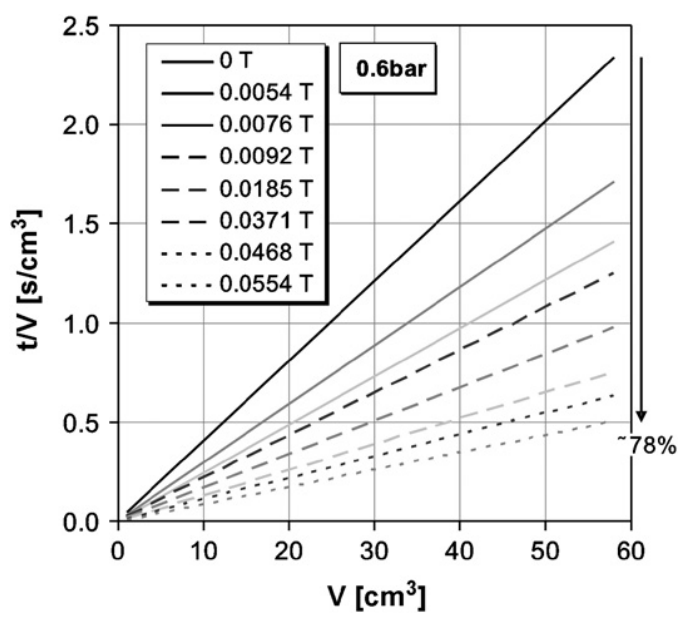

Fig. 10. $t / V$ vs. $V$ curves for different flux densities for 0.6 bar.

where a limit of $E_{S} \approx 0.25$ is reached after $20 \mathrm{~s}$ (Fig. 9). In the course this value even drops to 0.07 . The enlarged view reveals clearly that for one applied flux density the filter cake is less structured for $1.8 \mathrm{bar}$ (open symbols) than for 0.6 bar (filled symbols). While for 0.6 bar no noteworthy change in $E_{S}$ can be observed for field strengths higher than $0.0371 \mathrm{~T}$, for $1.8 \mathrm{bar}$ this limit is reached only for $0.0554 \mathrm{~T}$, because the hydrodynamic forces work against the alignment of the particles. But the minimum structuring parameter $E_{S}$ seems to be pressure independent.

To quantify the reduction of the filter cake resistance an overall evaluation of each run with Ruth's law is carried out (Eqs. (6) and (9)). In Figs. 10 and 11 the results are plotted in the form of the reciprocal flow rate $t / V_{l}$ versus filtrate volume $V_{l}$. Since the slope $a$ of the linear curves is proportional to the specific cake resistance, the influence of the $B$-field on the filtration resistance is directly visible. For 0.6 bar an acceleration of the cake building kinetics by $\approx 78 \%$ and for 1.8 bar by $\approx 62 \%$ is achieved superposing a magnetic field with $B=0.0554 \mathrm{~T}$. Again due to stronger hydrodynamic forces the positive effect of the magnetic field is lower for higher filtration pressures.

Fig. 12 gives a more detailed view of the change in the overall filter cake resistance. It shows the decreasing slopes from Eq. (9) as a function of the magnetic flux density for both differential pressures. Noticeable is the strong change in slope for low field strengths. This drop of resistance can be attributed to the alignment of 


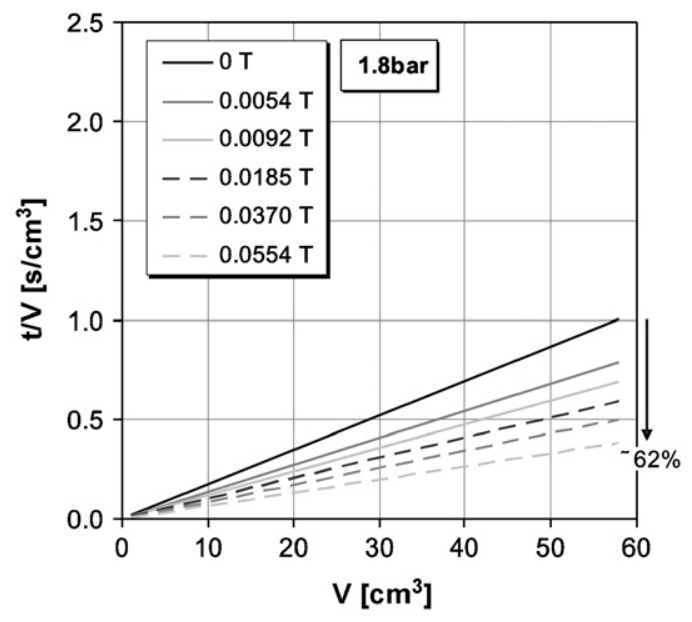

Fig. 11. $t / V$ vs. $V$ curves for different flux densities for 1.8 bar.

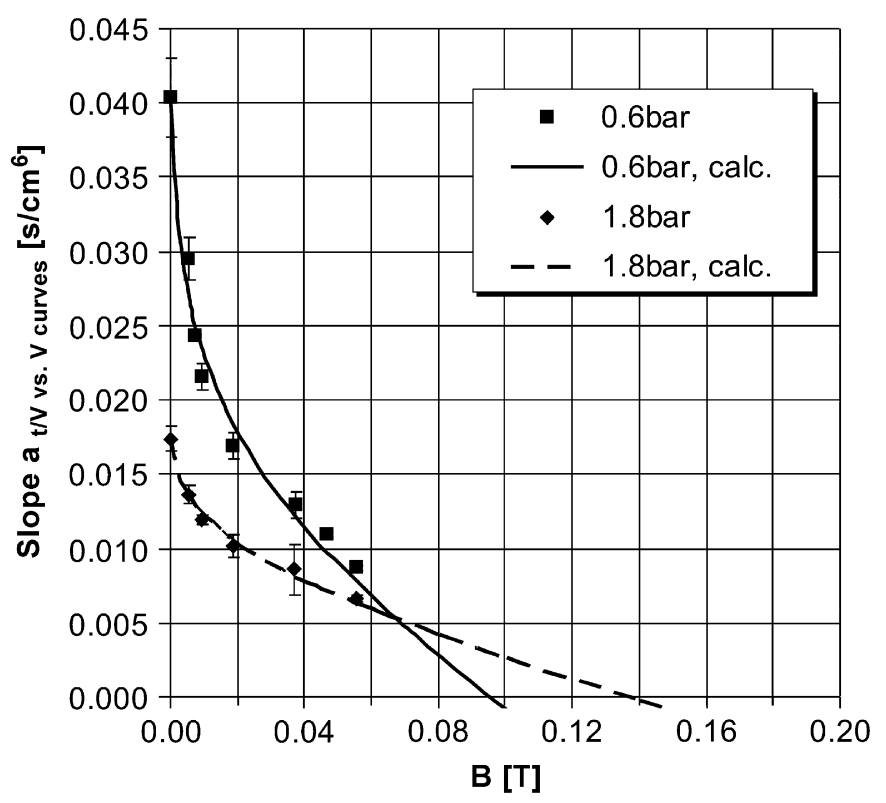

Fig. 12. Change of slope of Eq. (9) as a function of the applied flux density for 0.6 and 1.8 bar.

particles in the filter cake. For higher field strengths the maximum structuring is reached and the influence of the external magnetic force exceeds particle interactions and the slowdown of cake builtup dominates the process. Extrapolating the linear relation for higher field strengths to the abscissa the critical flux density $B_{\text {crit }}$ can be determined experimentally.

The lines in the plot give calculated results, when assuming a porosity function as stated in Eq. (12). Therefore the average particle distance in classical filtration $r_{0}$ and the minimum repulsive potential $E_{\min }$ are fitted to the experimental results for each pressure step using the extrapolated values of $B_{\text {crit }}$ for the magnetophoretic coefficient. With the average distance between the particles in classical filtration of $r_{0}=0.82 \mu \mathrm{m}$ for $0.6 \operatorname{bar}\left(r_{0}=0.72 \mu \mathrm{m}\right.$ for $\left.1.8 \mathrm{bar}\right)$ a porosity of 0.35 for 0.6 bar ( 0.33 for 1.8 bar) is calculated. Due to the magnetic structuring of the filter cake the porosity increases up to the limit of 0.46 for 0.6 bar ( 0.39 for 1.8 bar).

To evaluate the values of $E_{\min }$ they are compared with calculated data (Table 2). The absolute maximum of the magnetic repulsive potential can be calculated with Eq. (4) assuming

Table 2

Repulsive magnetic potentials

\begin{tabular}{lllll}
\hline & $M$ & $r$ & $\Delta p=0.6$ bar & $\Delta p=1.8$ bar \\
\hline$E_{m, \text { max }}(\mathrm{J})$ & $M_{S}$ & 0 & $2.7 \times 10^{-15}$ & \\
$E_{m, r 0}(\mathrm{~J})$ & $M_{S}$ & $r_{0}$ & $1.6 \times 10^{-15}$ & $1.7 \times 10^{-15}$ \\
$E_{\min }(\mathrm{J})$ & $M$ & $>r_{0}$ & $4.1 \times 10^{-18}-7.3 \times 10^{-16}$ & $4.4 \times 10^{-18}-9.1 \times 10^{-16}$ \\
\hline
\end{tabular}

direct particle contact and $M=M_{S}$. But in fact direct particle contact has to be negated. Thus more realistic values can be calculated incorporating the particle distance $r_{0}$ and $M=M_{S}$.

Comparing the fitted values of $E_{\min }$ to the possible maximum a difference of one to three decades can be found. Since in all experiments the magnetization does not reach saturation and the distance between the particles is larger than $r_{0}$ to reduce the repulsive potential, these values seem physically reasonable. All in all the calculated and the experimental results are in good agreement and describe the influence of the two different effects of the magnetic field sufficiently. The deviations are mainly due to the assumptions of the columnar particle alignment, the field strength invariant interactions between the particle chains and a non-perfectly homogeneous field distribution.

\section{Conclusions}

The hybrid process magnetic field enhanced cake filtration is shown to be highly efficient for the separation of superparamagnetic nanocomposites. Initially interparticle magnetic forces cause structured cakes with higher permeability which is confirmed by the structure parameter $E_{S}$. For higher field strengths a maximum improvement due to the aligned particles is reached and the external magnetic force which reduces the velocity of cake building leads to further improvement of the filtration kinetics. For each differential pressure one critical field strength exists above which no filter cake is formed and the filtrate accumulates abruptly. Calculations of the filtration process regarding both effects of the magnetic field individually are in good agreement with the experimental results. The new process offers high potential for selective bio-separation in the downstream processing. The whole process can be integrated in one single unit operation. Due to their superparamagnetic character the carrier particles can be used for several cycles.

\section{Notation}

$a$

A

$b$

B

$c_{v}$

$d$

E

$E_{S}$

F

H

$K^{*}$

$m$

M

$p$

$P^{*}$

$r$

$r_{C}$

$R$

$R_{m}$ coefficient in Eq. (6), s/m $\mathrm{m}^{6}$

filter area, $\mathrm{m}^{2}$

coefficient in Eq. (6), s/m $\mathrm{m}^{3}$

magnetic flux density, $\mathrm{T}$

volume concentration, dimensionless

diameter, $\mathrm{m}$

potential, J

structure coefficient, dimensionless

force, $\mathrm{N}$

magnetic field strength, $\mathrm{A} / \mathrm{m}$

coefficient in Eq. (9), dimensionless

mass, $\mathrm{g}$

magnetization, $\mathrm{A} / \mathrm{m}, \mathrm{A} \mathrm{m}^{2} / \mathrm{kg}$

pressure, bar

coefficient in Eq. (9), dimensionless

distance, $\mathrm{m}$

specific cake resistance, $\mathrm{m}^{-2}$

filtration resistance, $\mathrm{m}^{-1}$

filter media resistance, $\mathrm{m}^{-1}$ 
$t \quad$ time, s

u velocity, $\mathrm{m} / \mathrm{s}$

$V \quad$ volume, $\mathrm{m}^{3}$

\section{Greek letters}

$\varepsilon$

$\eta$

$\mu_{0}$

$\mu_{r}$

$\xi$

$\rho$

$\phi$

$\chi$

Indices

$\begin{array}{ll}0 & \text { classicalfiltration } \\ \text { crit } & \text { critical } \\ \text { drag } & \text { drag/hydrodynamic } \\ l & \text { liquid } \\ m & \text { magnetic } \\ \max & \text { maximum } \\ \min & \text { minimum } \\ p & \text { particle } \\ r & \text { remanent } \\ s & \text { saturation }\end{array}$

\section{Acknowledgments}

The authors owe special thanks to the Research Center Karlsruhe, Department of Water and Geotechnology (PD Dr.-Ing. M. Franzreb, Dipl.-Ing. M. Silvestre), for the contribution of the superparamagnetic nanocomposites. With their help and the help of the BMWi, Bokela $\mathrm{GmbH}$ and Steinert Elektromagnetbau $\mathrm{GmbH}$ magnetic filtration can be investigated further.

\section{References}

Bergmann, L., Schaefer, C., 2006. Elektromagnetismus, Band 2. In: Raith, W. (Ed.), Lehrbuch der Experimentalphysik, 9. Berlin, de Gruyter, Berlin (in German).
Butler, R., Banerjee, S., 1975. Theoretical single-domain grain size range in magnetite and titanomagnetite. Journal of Geophysical Research 80 (29), 4049-4058.

Charles, S., 1988. Aggregation in magnetic fluids and magnetic fluid composites. Chemical Engineering Communications 67, 145-180.

Chin, C., Yiacoumi, S., Tsouris, C., Relle, S., Grant, S., 2000. Secondaryminimum aggregation of superparamagnetic colloidal particles. Langmuir $16(8)$ $3641-3650$.

Chin, C., Yiacoumi, S., Tsouris, C., 2001. Probing DLVO forces using interparticle magnetic forces: transition from secondary-minimum to primary-minimum aggregation. Langmuir 17 (20), 6065-6071.

Dubowik, J., 1996. Shape anisotropy of magnetic heterostructures. Physical Review B 54 (2), 1088-1091.

Eichholz, C., Stolarski, M., Fuchs, B., Nirschl, H., 2007. Magnetfeldüberlagerte Pressfiltration. Chemie Ingenieur Technik 79 (4), 416-420 (in German).

Franzreb, M., Höll, W., 2000. Phosphate removal by high-gradient magnetic filtration using permanent magnets. IEEE Transactions on Applied Superconductivity 10 (1), 923-926.

Franzreb, M., Siemann-Herzberg, M., Hobley, T., Thomas, O., 2006. Protein purification using magnetic adsorbent particles. Applied Microbiology and Biotechnology 70 (5), 505-516.

Fuchs, B., 2005. Die Magnetfeldüberlagerte Kuchenfiltration, Ein neues hybrides Separationsverfahren. Dissertation. Universität Karlsruhe(TH), Shaker, Aachen, (in German).

Fuchs, B., Stolarski, M., Stahl, W., Nirschl, H., 2006. Magnetic field enhanced cakefiltration. Filtration 6 (4), 333-339.

Lee, W., Jovanovic, G., Kim, H., 1999. The effect of interparticle forces on fluidization regimes in the magnetized fluidized beds. Korean Journal of Chemical Engineering 16 (3), 362-370.

Nusser, M., 2007. Biotechnology in Germany-Employment Potentials and International Competitiveness. In: BIOforum Europe - Trends\&Techniques in Life Science Research 11 (7/8), 8-10.

PricewaterhouseCoopers, 2007. Pharmaceutical Sector Insights 2006/2007. Analysis \& opinion on global M\&A activity from our network of local advisers.

Rosensweig, R., Jerauld, G., Zahn, M., 1981. Structure of magnetically stabilized fluidized solids. Continuum Models of Discrete Systems 4, 137-144.

Ruth, B., Montillo, G., Montonna, H., 1933. Studies in filtration, II. Fundamental axiom of constant-pressure filtration. Industrial Engineering Chemistry 25 (2) 153-161.

Safarik, I., Ptackova, L., Safarikova, M., 2001. Large-scale separation of magnetic bioaffinity adsorbents. Biotechnology Letters 23, 1953-1956.

Sorrentino, J., 2002. Advances in correlating filter cake properties with particle collective characteristics. Dissertation, Universität Karlsruhe (TH), Shaker Aachen.

Stiess, M., 1995. Mechanische Verfahrenstechnik, 2. Band 1 und 2. Springer, Berlin. (in German).

Stolarski, M., Fuchs, B., Bogale, S., Eichholz, C., Nirschl, H., 2006. Magnetic field enhanced press-filtration. Chemical Engineering Science 61 (19), 6395-6403.

Stolarski, M., Eichholz, C., Fuchs, B., Nirschl, H., 2007. Sedimentation acceleration of remanent iron oxide by magnetic flocculation. China Particuology 5, 145-150.

Svoboda, J., 1987. Magnetic Methods for the Treatment of Minerals. In: Developments in Mineral Processing, vol. 8, Elsevier, Amsterdam. 